\title{
Research on Development and Model of Intelligent Transportation Systems
}

\author{
Wang Danping ${ }^{1,2,3}$, Hu Kunyuan ${ }^{1}$, Han Xiaowei $^{2}$ \\ ${ }^{1}$ Shenyang Institute of Automation Chinese Academy of Sciences, 110016 Shenyang, China \\ ${ }^{2}$ Shenyang University, 110044 Shenyang, China \\ aiamdanping@163.com
}

Keywords: Intelligent Transportation System; Architecture; Model-designing

\begin{abstract}
With the rapid development of economy and society, Intelligent Transport Systems also known as the road traffic information communication system, based on systems engineering, electronics, communications, information, and other high-tech, and have penetrated into the new transportation system of aviation, shipping, rail transport sector. In this paper, there is the basic organizational framework for intelligent transportation, intelligent transportation network proposed model and its data storage structure, and the important influence on optimal path trajectory intelligent transportation planning. This paper analyze intersection road network in the distribution based on computer. Establish ITS can improve after the road network line capacity and service levels, improve environmental quality and improve energy efficiency. There are introduction and overview of the basic concepts of domestic and international development, and proposed disciplinary system model of intelligent transportation systems, and network services model hierarchical model, and a brief analysis a model of meaning. Finally, the system standardization issues and model of the role of standardization briefly introduced.
\end{abstract}

\section{Introduction}

Intelligent Transportation is the future direction of the transport system, it is the effective integration of advanced information technology, data communications transmission technology, electronic sensor technology, control technology and computer technology, which are applied to the entire surface of a traffic management system and the establishment of in a wide range, full play to the role of real-time, accurate and efficient integrated transportation management system intelligent Transportation has the following two characteristics: First, focus on a wide range of applications and services traffic information, the second is focused on improving the existing traffic operating efficiency of facilities..

Compared with the general technology systems, intelligent transportation systems integrity requirements of the process is more stringent. Such integrity is reflected in: Intelligent transportation system construction involves many industries, is a complex system engineering giant community-wide participation. Intelligent Transportation Systems has integrated traffic engineering, information engineering, control engineering achievement, communication technology, computer technology, and many other scientific fields, require many skilled work together. Government, enterprises, research institutes and universities to participate in, locate the appropriate roles and tasks share, which is an important prerequisite for effective system expanded. Intelligent Transportation Systems will be dominated by mobile communications, broadband networks, RFID, sensors, cloud computing and other new generation of information technology for support, more in line with application requirements, improve the degree of trustworthiness and become ubiquitous [1].

Intelligent transportation system construction must be based on intelligent transportation systems framework. Framework of intelligent transportation system is mainly based on the needs of users in a geographical area, the definition of system functionality, content and methods of information exchange between functional domains, each function processing, etc., for all levels of information sharing, exchange and co-processing specification, is a qualitative description of the intelligent 
transportation system is to ensure that the process of coordination and technical integration architecture construction and implementation of intelligent transportation systems.

The 21st century will be the century of intelligent transportation, and people will be using the Intelligent Transportation System, which is an advanced integrated transport integrated management system. In this system, a vehicle travel is on their own intelligence freedom on the road, the road traffic is on its own intelligence will be adjusted to the optimum state. Intelligent transportation is based on modern electronic information technology systems for the transportation service. Its salient features are information collection, processing, dissemination, exchange, analysis, use of the main line, to provide transportation services for the diversity of the participants.

\section{Needs analysis of Intelligent Transportation Systems}

Intelligent Transportation Systems are currently accepted to fully and effectively alleviate the transport sector to solve problems, especially traffic congestion, one of the best ways to traffic jams, accidents, and traffic pollution and a series of traffic problems. The so-called intelligent transportation systems, is to people, vehicles, road and environment through communication, information, and other high-tech means to organically combine to achieve road safety, smooth, low pollution and low power consumption of software systems is a promotion of harmony between man and the environment of road transport integrated technology development [2].

ITS is based on the theory of dynamic traffic assignment, real-time traffic conditions on the road network to analyse complex, integrated use of 3G technology, advanced communications and computer technology. Currently appear overwhelming concept of things, in the face of real-time urban traffic flow guidance system complexity can be considered through the use of Internet of Things technology to achieve secondary traffic flow guidance system, the perfect solution to promote traffic-induced problems. The use of on-board computer, radio and other facilities dynamic, real-time optimal path to provide guidance to travellers instruction and real-time traffic information, and ultimately achieve the purpose of balancing network traffic flow, thereby reducing the length of stay of vehicles on the road, effectively preventing traffic congestion occurs, and ultimately achieve optimal allocation of traffic on each link in the network.

This paper briefly discusses Internet of Things based urban intelligent traffic flow guidance system and the needs of the system analysis and system functions and so on. The goal of building this system are: establishment of the Internet of Things system based on traffic flow guidance, a user through the car system shortest path analysis, geographic information query, vehicle location and bus route search functions, real fast, green, safe, and comfortable traffic environment and promote sustainable development of cities [3]. Fig.1 shows the intelligent traffic under Internet of Things. Fig. 1 shows the relationship between the data and the attributes of the description.

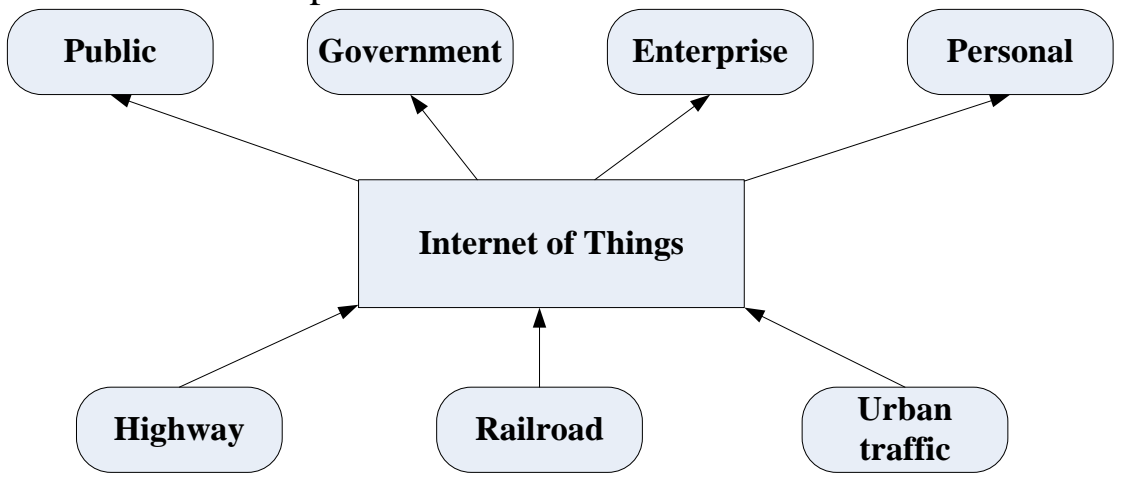

Fig. 1.The intelligent traffic under Internet of Things

\section{Design of vehicle static optimal path}

Static optimal path of the vehicle use the inherent static map data, which is stored in the on board systems, query the whole story optimal path algorithm road intersection nodes. The research on the static optimal path is not only the main road route search method autonomous vehicle navigation 
information system; also intelligent transportation systems, dynamic route planning based on the centre-based vehicle navigation system, real-time dynamic traffic information. The intelligent transportation-related scientists mainly focus on an algorithm that compares the corresponding theoretical and experimental results on the same data structure, contrary to the concept of statistics and logic. Since the memory data structures and intelligent transportation route planning digital map of the relationship between the efficiency is directly related to the timeliness and reliability of

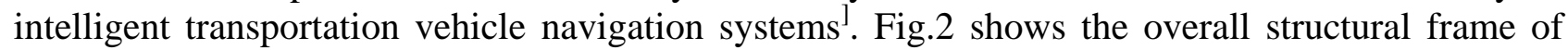
simulation system.

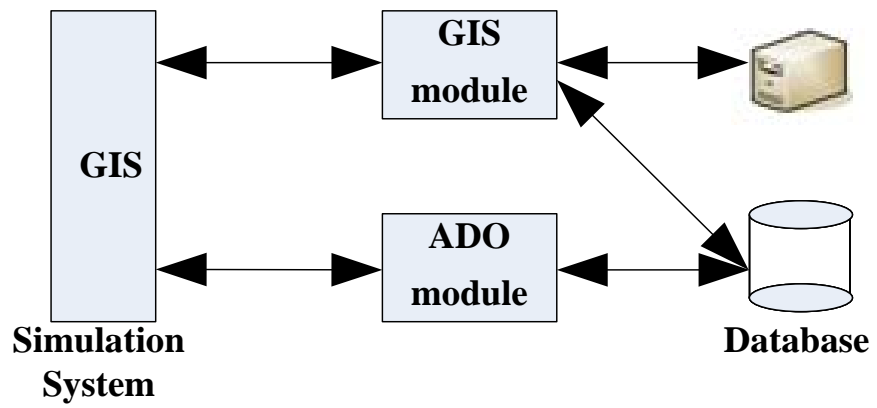

Fig. 2.The overall structural frame of simulation system

Optimal vehicle path planning simulation platform design and development, the GIS system development is divided into two layers: one layer of general information, development of the lower unity, its design ideas in line with ISO international software development standards; second is common information platform based on the expansion of secondary development function, for commercial geographic information systems. Data Flow System software development framework, mainly around the First Map X geographic information systems for intelligent transportation road network digital electronic map display, and the road junction node and path-related inquiries space on; Second, through ADO control basic electronic map metadata query, metadata describes these basic electronic classified information, and electronic map to subdivide property database query information [5]. Fig.3 shows the procedures display interface of simulation platform.

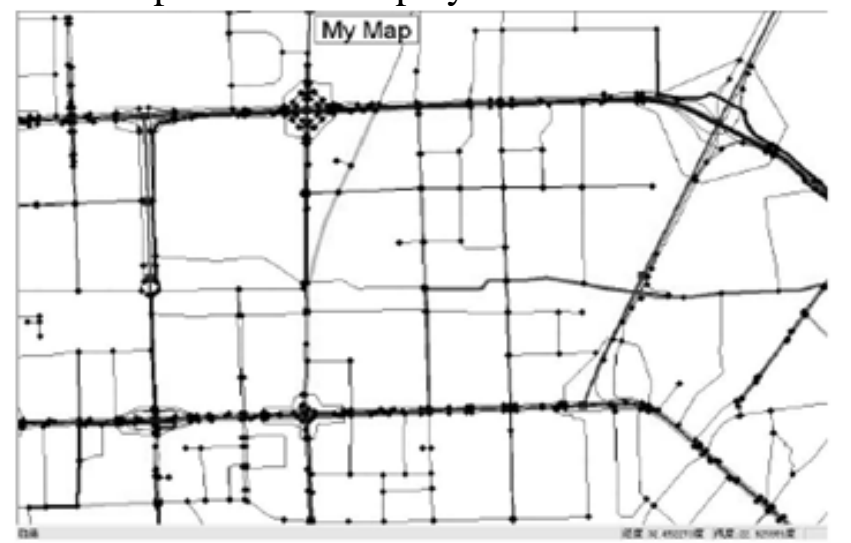

Fig. 3.The procedures display interface of simulation platform

The stepper motor is an electrical pulse signal is converted to angular displacement or linear displacement of electromechanical DAC, stepper motor working principle is based on the stator with energized electromagnet attracts the armature optional turn generate torque and rotation that rely on The electromagnetic magnet gravitational energy is converted into mechanical angular displacement. In the case of non-overloading, motor speed, stopped position depends only on the number of pulse frequency and pulse signals, without the impact of varying load, namely a pulse signal applied to the motor, the motor is turned one step angle. The number of pulses determines the size of the corner, and the frequency of pulses determines the motor speed.

Traffic data analysis and mining, which according to the traffic management and traveller access to real-time traffic information and intelligence requirements to dynamic traffic and road network congestion status-oriented analysis, combined with spatial and temporal characteristics of data traffic and transportation constraints, depth analysis of the potential similarities between correlation 
and correlation data, and traffic data clustering analysis, predictive analysis, correlation analysis, anomaly detection depth of excavation, to discover different characteristics dimensions, different data granularity implicit knowledge provide decision support for traffic management and traveller. Traffic data analysis and data mining is the core of traffic analysis. Temporal data analysis is usually for specific areas or for a specific problem. This article is for intelligent transportation, demand for dynamic analysis of traffic flow and road network congestion status to the road network and moving objects as the main basis of analysis the road network topological and temporal characteristics of the traffic flow on the design concept model of dynamic transportation network, integrated spatial data management and streaming data management technology, multi-temporal data granularity hierarchical organization and management, support for user access to the track, traffic, and status of different data elements [6].

\section{Design of vehicle dynamic optimal path}

Intelligent Transportation road network, on one side, the road traffic information can be collected feedback information to make the right road traffic surrounding circumstances, the vehicle can be dynamic optimal path planning, on the other side, real-time traffic information can't be effectively around feedback, travel information management system of the vehicle, the vehicle can only be static path planning of road-vehicle navigation systems; which is the study of scientific research in the field of intelligent traffic engineering hot issues. The Intelligent Transportation Systems are the primary means of solving traffic problems. Vehicle dynamic path planning is more than the static path planning process to reflect the status of the vehicle's credibility, timeliness and accuracy [7]. Fig.4 shows the overall power circuit.

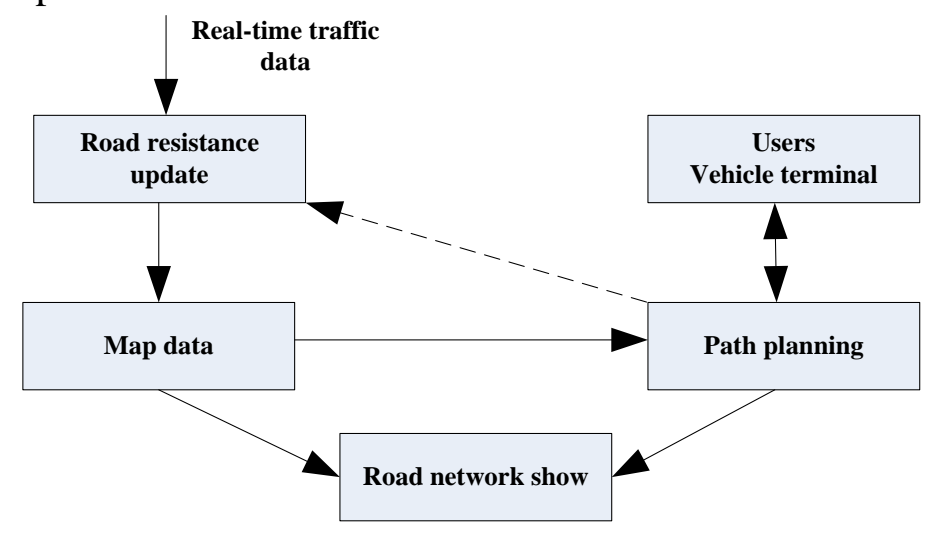

Fig. 4.The overall power circuit

Travellers issue needs to transport network central control system for a vehicle route planning, central process control system based on real-time road traffic information needs, determine the transportation network, dynamic programming the optimal path travelled by the vehicle, and transmit data to the onboard navigation path road network display module. Intelligent transportation network road vehicle display module can set the display by the vehicle traffic around the beginning and end nodes on the network map; and it is able to display the route planning and control system, the calculated optimal path.

Vehicle dynamic path optimization system use the flow of traffic from the traffic information and integrated processing platform combining network topology information, the right travel time is calculated based on the weight of the road, and then use the appropriate algorithm to improve the path optimization. The dynamic traffic equilibrium dynamic optimization uses the information systems to optimize the system for the traveling staff provide guidance information, while providing a balanced traffic guidance strategy for urban transport, information and guidance strategy to induce the dynamic traffic flow equalization system and constitutes the feedback information [8]. 


\section{Model-designing \& architecture}

In the case of the wide application of networking technology, the field of intelligent transportation systems of the services provided broader. The establishment of a network of intelligent transportation system services model based on the application of materials, through the analysis of different types of service users in all aspects of demand and transportation systems available, can better play networking technology and intelligent transportation systems, and improve infrastructure utilization.

ITS system control centre is responsible for traffic control, vehicle identification, vehicle parked integrated management, public transport time planning, urban transport planning and traffic management based on statistical knowledge; at the application level is divided into three areas: application services, building maintenance management and operational control.. In the application service road traffic information centre to collect information, real-time weather conditions, the schedule also includes public vehicles, road network of digital maps and some route guidance algorithm under certain conditions, transferred to the management and traveller information service needs, important part of the operation control embodies the characteristics of Things intelligent transportation systems, the core part of that feedback and control information, focusing on scheduling, transportation cargo transportation route planning behaviour, and safety of the vehicle and the operating environment, higher demands.

ITS first is based on road infrastructure, including road network itself and the auxiliary equipment such as beacons, roadside sensors, which belong to the physical layer; ITS emphasized sharing information and take advantage of, in which the flow of information and transmitted by the transmission layer to complete, including the short-range microwave communications, satellite, optic communications and the like; treatment layer is responsible for completing the extraction and processing of information, to generate a database of various kinds of data, and can provide different requirements for different services or different sectors of information; intelligent layer includes expert knowledge of system statistics such as public transport scheduling, decision support, such as guidance and automatic path planning, adaptive processing based on real-time traffic flow information such as traffic lights and other functions based on the adjustment; the one hand, according to the intelligence service layer provide functionality for different service areas, on the other hand, depending on the service requirements for continuously extended functional intelligent layer. Fig.5 shows the Network-level model of ITS.

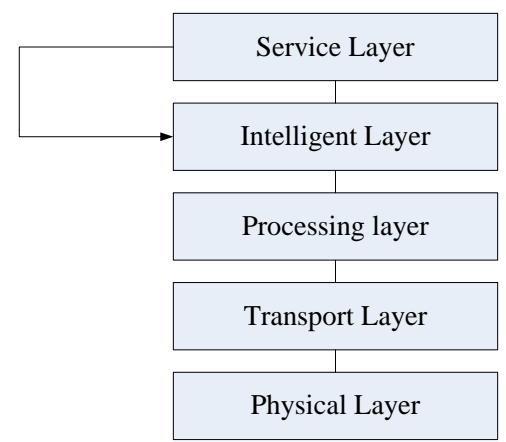

Fig. 5.The Network-level model of ITS

Basic level is the perception layer networking technologies in intelligent transportation application, and it provides an efficient means of information exchange technology for top job control and business management, and physical events and data collection of traffic environment for the entire transportation system. In the perception layer is divided into data acquisition and sensor networking information layer co-processing of two parts. Network layer can achieve a more widespread application of Internet capabilities in intelligent transportation systems, and perceive the information accessible, efficient and secure transfer of information, and therefore need to sensor networks and mobile communication technology, Internet technology integration. Due to the need for intelligent transportation systems and perceived end-aware network node address management identified and resolved, so the network layer of the Internet of Things should provide the 
appropriate information resources management and storage technologies, M2M wireless access and remote control technology in order to achieve things direct intelligent control things and things in between.

Application layer is based on traffic data resources collected by the perception layer into a platform for intelligent transportation systems, the formation of various transport subsystem application specification data through a unified standard, used in supporting platform and application services, including data interface used to support cross-industry, cross-application, cross system collaboration between the information sharing, interoperability features. Things data in support of the main applications of intelligent transportation system is divided into four modules: traffic management systems, customer service systems, traffic management systems, traffic control systems [10].

Through networking technology to build the initiative to contact intelligent traffic information system platform and the vehicle terminal, abandon past passively collected information form, information and vehicle information platform for two-way communication between the terminals. Intelligent traffic control system will be based on information collected from the vehicle and traffic information, combined with computer control technology and intelligent algorithm analysis, complete a comprehensive perception of roads and transport, in order to achieve effective regulation of traffic information, and provide convenient services for vehicle users, to achieve full-time traffic control, provide transportation efficiency and traffic safety based networks and applications, making the traffic management in anticipation beforehand disposal into the active management model, but also profound changes in the field of intelligent transportation management system.

\section{Conclusions}

As an integral part in the industry chain, intelligent transportation has the characteristics that is highly used in the application market, maturely technological and greatly supported by government, therefore, the introduction of Internet of Things will dramatically change the service model and system architecture of ITS, and the market prospect is broad. The dynamic mathematical model of single vehicle is used vehicle planning algorithm based on period, effectively avoided the traffic road, reduce vehicle travel cost, improve the real-time effect and accuracy of the vehicle dynamic path. We hope the results and researches could combine with reality in order to reduce traffic congestion. Meanwhile, traffic network optimization has been realized to reduce traffic congestion areas. This paper has optimized regional traffic signal control system, traffic guidance as well as traffic assignment, involved data sources, data collection as well as the relationship between guidance obeisance rate and traffic jam.

\section{Acknowledgements}

The research work was supported by Liaoning city of Shenyang province science and technology plan project number: F13-298-1-00.

\section{References}

[1] David Levinson. The Value of advanced traveller information systems for route choice, Transportation Research Part C 11(2003):75-87.

[2] G. A. Giannopoulos The application of information and communication technologies in transport. European Journal of Operational Research 152(2004):302-320.

[3] FU L P, L RILETT R. Expected shortest paths in dynamic and stochastic traffic networks [J], Transportation Research B, 2008, 32(7): 499-516. 
[4] Xia Ying, Hong Mei Gan, Gyoung Bae Kim. Design and Implementation of Advanced Traffic Monitoring System Based on Integration of Data Stream Management System and Spatial DBMS[J]. Journal of Korea Spatial Information System Society, 2009, 11(2): 162-169.

[5] Gonzalez H, Han Jia-lei. Adaptive fastest path computation on a road network: a traffic mining approach [C] Process of the 33rd International Conference on Very Large Data Bases. Austria: VLDB Press, 2007:23-28.

[6] Oje Kwon, Yong-Soo Song, Jae-Hun Kim, Ki-Joune Li. SCONSTREAM: A Spatial Context Stream Processing System[C]. International Conference on Computational Science and Its Applications (ICCSA), 2010: 165-170.

[7] LEE T K, PARK S, HUANG Z, et al. Toward Seoul road sign management on LarKC platform [C]//Proceedings of the 9th International Semantic; Web Conference (ISWC2010), Track of Posters and Demos ,2010.

[8] Wang Yu-Ping, The Theory and Method of Evolutionary Computation, Beijing: Science Press, 2011.

[9] LI N, HUANG Z, XU D, et al. Interface Design of Semantic; System for Road Sign Management [C], Web Information Systems Engineering-WISE 2013 Workshops Springer Berlin Heidelberg, 2014:452-460.

[10] WANC D, HUANG Z, LIU Q, et al. Using Semantic Technology for Consistency Checking of Road Signs [C]. Web Information Systems Engineering-WISE 2013 Workshops Springer Berlin Heidelberg, 2014:452-460. 